\title{
The human microbiome and genetic disease: towards the integration of metagenomic and multi-omics data
}

\author{
Huiying Zhao ${ }^{1}$ \\ Published online: 30 March 2021 \\ (c) The Author(s), under exclusive licence to Springer-Verlag GmbH Germany, part of Springer Nature 2021
}

The microbiome is increasingly recognized as having a fundamental role in human physiology, in the context of both health and disease (Rothschild 2018). Indeed, the composition of the microbiota has been found to be a key factor associated with a variety of human genetic diseases. For example, in several neurological disorders, such as Alzheimer's disease, Parkinson's disease and stroke, the microbiome has emerged as an important player in disease causation and modulation (Sampson 2020; Sgritta 2019). Similarly, in cancer, the microbiome is now recognized as playing key roles in inflammation, the immune response and the generation of toxic metabolites, thereby contributing to the process of carcinogenesis (Coker 2018; Dejea 2018; Tanoue 2019). The rapid evolution of sequencing technology and increases in study sample sizes have brought in their wake new developments such as microbiome-wide association studies designed to characterize the microbiota and their impact on different human diseases (Gilbert 2016; Wang 2018).

Various attempts have been made to characterize the influence of the human microbiome in different tissues and at different stages in disease pathogenesis by integrating microbiome data, specifically metagenomic data, with multiomics data (Liu 2020; Integrative 2019). Metagenomic data have frequently been integrated with metatranscriptomic, metaproteomic and metabolomic data to quantify microbiome abundance in terms of gene expression, and protein and metabolite levels (Narayanasamy 2016; Nies 2021; Martinez Arbas 2021). The integration of multi-omics data has provided novel insights into microbiome functions by increasing the complexity of the data available, which has in turn stimulated the development of novel bioinformatics and statistical approaches. A recurring theme in current

Huiying Zhao

zhaohy8@mail.sysu.edu.cn

1 Department of Medical Research Center, Sun Yat-Sen Memorial Hospital, Sun Yat-Sen University, Guangzhou, China microbiome studies is the application of efficient analytical approaches to discovering novel relationships between the microbiome and human genetic diseases. These studies serve to improve our understanding of the mechanisms by which the microbiome influences human genetic disease at multiple levels. Furthermore, they have enabled a shift from investigating disease causation to using these data in diagnosis and in the design of novel therapeutics. In this issue, we present four articles fitting the theme "The human microbiome and genetic disease", which together exemplify the exploration of human genetic disease through gut microbiome studies, and highlight new approaches as well as novel findings in relation to disease mechanisms.

The gut microbiome influences host health by operating as a regulator of host pathways for diseases, and consequently affecting immune systems and energy metabolism. In their review, Nichols and Davenport (2020) describe studies of the relationship between the microbiome and the host transcriptome that have been performed using model organisms, by collecting biopsies, or generating organoids. To better understand the relationship between the microbiome and the host, single-cell sequencing and organoid systems are proposed as future technologies to link a host with the microbes living within it (Nichols and Davenport 2020). Evidence has also accumulated to indicate the roles of the microbiome in tumorigenesis (Sanchez-Alcoholado 2020; Bhatt et al. 2017; Helmink et al. 2019). The second article, by Mima et al., explores the integration of microbiology into the molecular pathological epidemiology model and provides a research framework for uncovering the roles of the microbiome in influencing the biology of both tumor cells and immune cells (Mima 2020). The gut microbiome has been found to play important roles in inflammatory bowel disease (IBD), and as Collij et al. show, has the potential to become both a diagnostic tool and a therapeutic option in IBD if setting a gold standard to establish sample collecting for biobanking (Collij et al. 2020). Finally, there is also a role for gut microbiota in influencing the outcome of solid 
organ transplantation. In this context, Qin et al. show that the alteration of the microbiome may contribute to the development of graft fibrosis after pediatric liver transplantation (Qin et al. 2020).

Our treatment of this special topic highlights some key advances made in interpreting human genetic disease through gut microbiome studies, emphasizing new approaches as well as novel findings in relation to disease mechanisms.

\section{References}

Bhatt AP, Redinbo MR, Bultman SJ (2017) The role of the microbiome in cancer development and therapy. CA Cancer J Clin 67:326-344. https://doi.org/10.3322/caac.21398

Coker OO et al (2018) Mucosal microbiomedysbiosis in gastric carcinogenesis. Gut 67:1024-1032. https://doi.org/10.1136/ gutjnl-2017-314281

Collij V, Klaassen MAY, Weersma RK, Vila AV (2020) Gut microbiota in inflammatory bowel diseases: moving from basic science to clinical applications. Hum Genet. https://doi.org/10.1007/ s00439-020-02218-3

de Nies L et al (2021) PathoFact: a pipeline for the prediction of virulence factors and antimicrobial resistance genes in metagenomic data. Microbiome 9:49. https://doi.org/10.1186/ s40168-020-00993-9

Dejea CM et al (2018) Patients with familial adenomatous polyposis harbor colonic biofilms containing tumorigenic bacteria. Science 359:592-597. https://doi.org/10.1126/science.aah3648

Gilbert JA et al (2016) Microbiome-wide association studies link dynamic microbial consortia to disease. Nature 535:94-103. https://doi.org/10.1038/nature 18850

Helmink BA, Khan MAW, Hermann A, Gopalakrishnan V, Wargo JA (2019) The microbiome, cancer, and cancer therapy. Nat Med 25:377-388. https://doi.org/10.1038/s41591-019-0377-7

Integrative HMPRNC (2019) The integrative human microbiome project. Nature 569:641-648. https://doi.org/10.1038/ s41586-019-1238-8

Liu J et al (2020) Integration of epidemiologic, pharmacologic, genetic and gut microbiome data in a drug-metabolite atlas. Nat Med 26:110-117. https://doi.org/10.1038/s41591-019-0722-x
Martinez Arbas S et al (2021) Roles of bacteriophages, plasmids and CRISPR immunity in microbial community dynamics revealed using time-series integrated meta-omics. Nat Microbiol 6:123135. https://doi.org/10.1038/s41564-020-00794-8

Mima K et al (2020) The microbiome, genetics, and gastrointestinal neoplasms: the evolving field of molecular pathological epidemiology to analyze the tumor-immune-microbiome interaction. Hum Genet. https://doi.org/10.1007/s00439-020-02235-2

Narayanasamy S et al (2016) IMP: a pipeline for reproducible reference-independent integrated metagenomic and metatranscriptomic analyses. Genome Biol 17:260. https://doi.org/10.1186/ s13059-016-1116-8

Nichols RG, Davenport ER (2020) The relationship between the gut microbiome and host gene expression: a review. Hum Genet. https://doi.org/10.1007/s00439-020-02237-0

Qin T, Fu J, Verkade HJ (2020) The role of the gut microbiome in graft fibrosis after pediatric liver transplantation. Hum Genet. https:// doi.org/10.1007/s00439-020-02221-8

Rothschild D et al (2018) Environment dominates over host genetics in shaping human gut microbiota. Nature 555:210-215. https://doi. org/10.1038/nature25973

Sampson TR et al (2020) A gut bacterial amyloid promotes alpha-synuclein aggregation and motor impairment in mice. Elife. https://doi. org/10.7554/eLife.53111

Sanchez-Alcoholado L et al (2020) The role of the gut microbiome in colorectal cancer development and therapy response. Cancers (Basel). https://doi.org/10.3390/cancers12061406

Sgritta M et al (2019) Mechanisms underlying microbial-mediated changes in social behavior in mouse models of autism spectrum disorder. Neuron 101(246-259):e246. https://doi.org/10.1016/j. neuron.2018.11.018

Tanoue $\mathrm{T}$ et al (2019) A defined commensal consortium elicits CD8 T cells and anti-cancer immunity. Nature 565:600-605. https://doi. org/10.1038/s41586-019-0878-z

Wang J et al (2018) Meta-analysis of human genome-microbiome association studies: the MiBioGen consortium initiative. Microbiome 6:101. https://doi.org/10.1186/s40168-018-0479-3

Publisher's Note Springer Nature remains neutral with regard to jurisdictional claims in published maps and institutional affiliations. 